BMJ Open Gastroenterology

\section{Colorectal cancer mortality 10 years after a single round of guaiac faecal occult blood test (gFOBT) screening: experiences from a Danish screening cohort}

To cite: Bjerrum A,

Andersen 0, Fischer A, et al. Colorectal cancer mortality 10 years after a single round of guaiac faecal occult blood test (gFOBT) screening: experiences from a Danish screening cohort. BMJ Open Gastro 2016;3:e000120. doi:10.1136/bmjgast-2016000120

Received 1 September 2016 Revised 3 October 2016 Accepted 18 October 2016

\footnotetext{
${ }^{1}$ Department of Public Health, University of Copenhagen, Copenhagen K, Denmark ${ }^{2}$ Copenhagen University Hospital Herlev, Herlev, Denmark

${ }^{3}$ Department of Pathology, Vejle Hospital, Vejle, Denmark

Correspondence to Dr Andreas Bjerrum; ab@medicinsk.dk
}

\section{ABSTRACT}

Background: In Denmark, colorectal cancer (CRC) is the third most frequent cancer. Randomised trials have shown that guaiac faecal occult blood test (gFOBT) screening can reduce CRC mortality, but a recent large randomised study from Finland did not find any effect. A feasibility study was carried out in Denmark in 2005-2006 where residents aged 50-74 years in 2 Danish counties were invited once to participate in gFOBT screening. We used the unique Danish registers to assess the impact of gFOBT screening in this group on CRC incidence and mortality.

Methods: In this cohort study, we followed a group comprising 166277 individuals invited to screening and a reference group comprising the remaining 1240348 Danes of the same age. We linked the Danish population and health service registers to obtain information about colonoscopies, polypectomies, incident CRC and cause of death. Results: After a median follow-up time of 8.9 years, the CRC mortality was significantly lower in the screening group than in the reference group with an adjusted HR (aHR) of $0.92(95 \% \mathrm{Cl} 0.86$ to 0.99$)$, while the aHR for all-cause mortality was $0.95(95 \% \mathrm{Cl}$ 0.94 to 0.96$)$. For screening participants, the aHR for CRC mortality and all-cause mortality was $0.72(0.64$ to 0.80 ) and 0.59 ( 0.57 to 0.60 ), respectively.

Conclusions: About 10 years after a single round of gFOBT screening, we found a significant $8 \%$ deficit in CRC mortality in the screening group compared with other Danes. We found almost the same deficit in allcause mortality, and on this basis, it is not possible to conclude that one screening round had an effect on CRC mortality. Our study indicated that close monitoring of the outcome of CRC screening is warranted.

\section{INTRODUCTION}

In Denmark, colorectal cancer (CRC) is the third most frequent cancer for men and women aged $50-74$ years with age-standardised incidences of 160 and 126 cases per 100000

\section{Summary box}

What is already known about this subject?

- Randomised controlled trials with faecal occult blood based screening have shown a reduction in colorectal cancer mortality.

- Based on the favourable outcomes, colorectal cancer screening is now widely implemented.

- However, no reduction in colorectal cancer mortality was found in a large Finnish randomised health service study, though with a relatively short follow-up time.

What are the new findings?

- We report long term follow-up from a Danish screening feasibility study including one round of faecal occult blood based screening.

- The population invited to screening had an $8 \%$ lower colorectal cancer mortality and a $5 \%$ lower all-cause mortality as compared with other Danes.

- As the deficits in colorectal cancer and all-cause mortality were almost equal, it is not possible to conclude that one screening round had an effect on colorectal cancer mortality.

How might it impact on clinical practice in the foreseeable future?

- Our study indicates that close monitoring of the outcome of colorectal cancer screening is warranted.

(Nordic Standard Population, 2007-2011). ${ }^{1}$ Several randomised trials have shown that guaiac faecal occult blood test (gFOBT) screening can reduce CRC mortality ${ }^{2-5}$ and CRC screening has been implemented in several countries to detect and treat CRC before the disease becomes symptomatic.

In a recent large randomised study from Finland, Pitkäniemi et $a l^{6}$ did not detect any effect of gFOBT screening on CRC mortality. 
In contrast to the previous randomised trials, ${ }^{7}$ the Finnish study was designed as a randomised health service study embedded in the routine health services and the authors argued that this was characterised by variation in available resources; a population that may have been less motivated; and health experts that were possibly not as devoted and did not follow guidelines as strictly as in scientific trials. ${ }^{6}$

As an input to the Danish debate on a possible national CRC screening programme, a feasibility study within the routine healthcare was carried out in 2005-2006. ${ }^{8} 9$ Residents aged 50-74 years in two Danish counties were invited once to participate in the screening performed with gFOBT. Colonoscopy or alternatively colonography was offered if the screening test was positive. From 1 January 2014, a nationwide screening programme was implemented in Denmark following the same scheme, but based on the faecal immunochemical test (FIT).

The unique Danish population and health registers allowed us to follow-up the population invited for the feasibility study for 10 years to assess the impact of the screening on CRC incidence and mortality in a routine healthcare setting.

\section{METHODS}

\section{Study design and study population}

In this population-based cohort-study, we included all Danish residents born between 3 August 1930 and 2 August 1955 and living in Denmark on 2 August 2005. The screening group comprised persons invited to CRC screening, that is, all inhabitants in Vejle county and a random sample of approximately half of the inhabitants in the Copenhagen county, while the reference group comprised the other Danes.

Individual invitation dates were known for the screening group. We used the invitation scheme from the screening group to assign pseudoinvitation dates to persons in the reference group. In the following, we use the term 'invitation date' for the 'true invitation dates' in the screening group and 'pseudoinvitation dates' in the reference group.

Based on unique personal identification, we linked each person with the Danish Cancer Register to obtain information about incident CRC cases, the National Patient Register for diagnoses and hospital procedures including colonoscopies and polypectomies, the Health Service Register for private sector colonoscopies and polypectomies, and the Death Cause Register.

We excluded persons who died or emigrated before the invitation date, were diagnosed with CRC (ICD10: C18/C19/C20/C21) or inflammatory bowel disease (ICD8: 563.01/563.02/563.09/563.19 and ICD10: K50/ K51) prior to invitation; or persons who underwent colonoscopy, sigmoidoscopy or any invasive colon or anorectal procedure in the period from 2 years before the screening programme started, that is, 2 August 2003, until the invitation date. Data linkage identified a number of persons excluded from screening for unknown reasons. These persons were excluded from the screening group in our analyses. We performed sensitivity analyses to assess the impact of this group on our results.

\section{Screening process}

Persons invited to the screening programme received a gFOBT kit per mail. The letter included an invitation describing the background and purpose of screening, instructions for sampling and a prepaid return envelope. Participants with a positive gFOBT were notified by mail and invited to colonoscopy or alternatively colonography.

In Vejle County, colonoscopies were performed at three selected hospitals. Diagnostic biopsy and polypectomy was performed as part of the follow-up procedure. In Copenhagen County, colonoscopies were performed in hospitals and private clinics. Patients with many adenomas were referred to hospitals for further diagnostic procedures and polypectomy. Postpolypectomy surveillance was not specified in the feasibility study, but undertaken according to the clinical practice at the respective hospitals.

\section{Definitions and statistical analyses}

Persons were followed from the invitation date to date of death, emigration or 31 December 2014, whichever came first. The first colonoscopy performed after a positive gFOBT was defined as the baseline colonoscopy, as

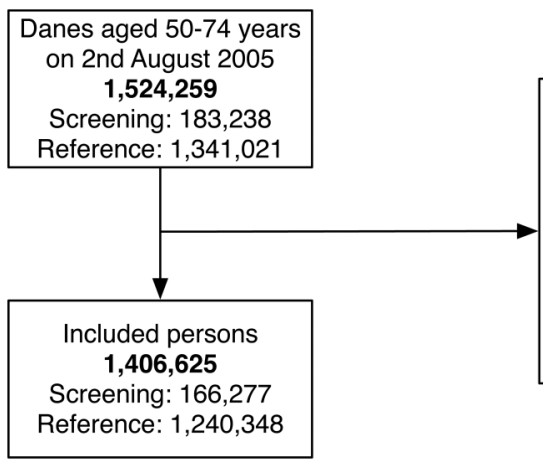

Total exclusions: 117,634

Dead before invitation: 18,311

Inflammatory bowel disease: 13,519

CRC before invitation: 12,328

Colonoscopy 2 years before invitation (public): 59,314

Colonoscopy 2 years before invitation (private): 25,074

Emigrated before invitation: 4,833

Excluded from screening for unknown reasons: 3,757

Figure 1 Inclusion and exclusion in the study population. ${ }^{1}$ The same person may fall for more than one criterion for exclusion. 
long as it was within 90 days of invitation. CRC-cases diagnosed within 180 days after the baseline colonoscopy were categorised as screen-detected CRC.

We calculated incidence-based CRC mortality rates for the screening and reference groups, including only CRC deaths in cases diagnosed after the invitation date. The multivariate Cox regression was used to calculate HRs adjusted for sex and age (aHR) for CRC incidence, GRC mortality and all-cause mortality.

\begin{tabular}{|c|c|c|c|}
\hline & $\begin{array}{l}\text { Screening } \\
\text { group }\end{array}$ & $\begin{array}{l}\text { Reference } \\
\text { group }\end{array}$ & $\begin{array}{l}p \\
\text { Value }\end{array}$ \\
\hline Population & 166277 & 1240348 & \\
\hline $\begin{array}{l}\text { Person time } \\
\text { (years) }\end{array}$ & 1393131 & 10358902 & \\
\hline \multicolumn{4}{|l|}{ Sex } \\
\hline Female & $48.9 \%$ & $49.5 \%$ & $<0.01$ \\
\hline Male & $51.1 \%$ & $50.5 \%$ & \\
\hline $\begin{array}{l}\text { Median age at } \\
\text { invitation }\end{array}$ & 60.6 & 60.5 & 0.06 \\
\hline $50-54$ years & $21.8 \%$ & $22.0 \%$ & 0.10 \\
\hline $55-59$ years & $25.2 \%$ & $25.4 \%$ & 0.08 \\
\hline $60-64$ years & $22.9 \%$ & $22.8 \%$ & 0.24 \\
\hline $65-69$ years & $16.7 \%$ & $16.3 \%$ & $<0.01$ \\
\hline $70-74$ years & $13.0 \%$ & $12.8 \%$ & $<0.01$ \\
\hline $74+$ years & $0.4 \%$ & $0.8 \%$ & $<0.01$ \\
\hline
\end{tabular}

The screening group includes persons invited to colorectal cancer screening in Vejle and Copenhagen counties. The reference group includes persons in the rest of Denmark in the same age group.
Gender-based analyses were adjusted for age, and persons in the screening group were compared with persons in the reference group of the same gender. We tested for interactions and proportionality based on Schoenfeld residuals.

We estimated the excess mortality rate by dividing the excess number of deaths observed in persons with CRC by the total number of person-years in the screening group and reference group (stratified by sex and age). The excess mortality rate ratio was calculated according to procedures described by van Leeuwen $e t a l .{ }^{10}$

All statistical analyses were conducted with Stata V.14.1.

\section{Ethics}

The Danish Data Protection Agency approved the linkage between the registers in this study (J.-no. 2015-41-4012). The study was not subject to approval by the Ethics Committee since data collection did not involve patient contact or access to patients' medical records.

\section{RESULTS}

We identified 1524259 persons aged 50-74 years and living in Denmark on 2 August 2005. A total of 117634 persons were excluded based on predefined criteria (figure 1). Consequently, this study included 1406625 persons divided into the screening group of 166277 , and a reference group of 1240348 . The median follow-up time was 8.9 years for the screening group and

Table 2 Comparison between the screening group from Vejle and Copenhagen counties and the intervention group from the randomised controlled trial in Funen, Denmark ${ }^{5}$

\begin{tabular}{|c|c|c|c|c|}
\hline & \multicolumn{2}{|c|}{$\begin{array}{l}\text { Screening group Vejle and } \\
\text { Copenhagen counties }\end{array}$} & \multicolumn{2}{|c|}{$\begin{array}{l}\text { Intervention group, randomised } \\
\text { controlled trial, Funen, Denmark }\end{array}$} \\
\hline & Number & $\%$ of group above & Number & $\%$ of group above \\
\hline Number invited & 166277 & NR & 30970 & NR \\
\hline Participants & 80563 & $48.5 \%$ & 20672 & $66.7 \%$ \\
\hline Positive FOBT & 1924 & $2.4 \%$ & 215 & $1.0 \%$ \\
\hline Colonoscopy & 1741 & $90.5 \%$ & 203 & $94.4 \%$ \\
\hline Colorectal cancer & 155 & $8.9 \%$ & 37 & $18.2 \%$ \\
\hline
\end{tabular}

Table 3 Percentage and number of persons with colonoscopies and polypectomy

\begin{tabular}{|c|c|c|c|c|}
\hline & \multicolumn{2}{|c|}{ Screening group } & \multicolumn{2}{|c|}{ Reference group } \\
\hline & No. & Percentage $^{\star}$ & No. & Percentage \\
\hline Persons with colonoscopy & 30264 & 18.2 & 206125 & 16.7 \\
\hline Persons with colonoscopy $\leq 2$ years from invitation & 7513 & 4.5 & 42886 & 3.5 \\
\hline Persons with colonoscopy $>2$ years from invitation & 25432 & 15.3 & 179282 & 14.5 \\
\hline Persons with polypectomy & 7157 & 4.3 & 46383 & 3.7 \\
\hline Persons with polypectomy $\leq 2$ years from invitation & 1808 & 1.1 & 8391 & 0.7 \\
\hline Persons with polypectomy $>2$ years from invitation & 5656 & 3.4 & 39364 & 3.2 \\
\hline
\end{tabular}


the reference group with an IQR of 208 and 210 days for the screening group and reference group, respectively. The longest follow-up was 9.4 years in both groups.

The proportion of women was marginally lower in the screening group than in the reference group (table 1). Moreover, in the screening group, there was a small but significant over-representation of persons aged 6574 years, and a marginally smaller proportion of persons aged 75 years and above.

In the screening group, 80563 persons (48.5\%) returned a stool sample. Among those, 1924 (2.4\%) had a positive gFOBT, of whom $1737(90.3 \%)$ underwent colonoscopy. Among those, 155 (8.9\%) patients were diagnosed with CRC (table 2). About $4.5 \%$ in the screening group underwent colonoscopy within the first 2 years after invitation compared with $3.5 \%$ in the reference group (table 3 ). More than 2 years after the invitation, the two groups had almost the same use of colonoscopies. Polypectomy was performed in $4.3 \%$ of the screening group compared with $3.7 \%$ in the reference group. Within the first 2 years after invitation, $1.1 \%$ from the screening group underwent polypectomy compared with $0.7 \%$ in the reference group.

The CRC incidence during the follow-up period was significantly lower in the screening group than in the reference group (aHR 0.94, 95\% CI 0.90 to 0.97) (table 4). In the screening group, CRC incidence rose quickly within the first year after invitation due to screen-detected cases. After $\sim 3.5$ years, the curve for the screening group crossed the curve for the reference group (figure 2).

The CRC mortality during the follow-up period was significantly lower in the screening group than in the reference group (aHR 0.92, 95\% CI 0.86 to 0.99 ), with the deficit coming from the Copenhagen part of the screening group (aHR for Vejle 0.96, 95\% CI 0.87 to 1.06; aHR for Copenhagen 0.89, 95\% CI 0.80 to 0.98 ) (table 4 ). We did not observe any difference between men and women. The all-cause mortality was also significantly lower in the screening group than in the reference group (aHR $0.95,95 \%$ CI 0.94 to 0.96 ), with the deficit coming from Vejle county (aHR for Vejle 0.91, 95\% CI 0.90 to 0.93; aHR for Copenhagen 1.00, 95\% CI 0.98 to 1.02). The deficit in Vejle was seen from the very beginning of the follow-up period (figure 3).

Perprotocol analysis showed that screening participants had lower CRC incidence (aHR 0.94, 95\% CI 0.90 to 0.97 ), lower CRC mortality (aHR $0.72,95 \%$ CI 0.64 to 0.80 ) and substantially lower all-cause mortality (aHR $0.59,95 \%$ CI 0.57 to 0.60 ) (table 4 ). Non-responders had significantly increased CRC mortality (aHR of 1.12, 95\% CI 1.03 to 1.23 ) and all-cause mortality (aHR 1.31, 95\% CI 1.29 to 1.33 ).

The excess mortality rate ratio due to CRC for the screening group was 0.93 (95\% CI 0.84 to 1.02 ); 0.93 (95\% CI 0.82 to 1.07 ) for Vejle County and 0.92 (95\% CI 0.80 to 1.06 ) for Copenhagen County.

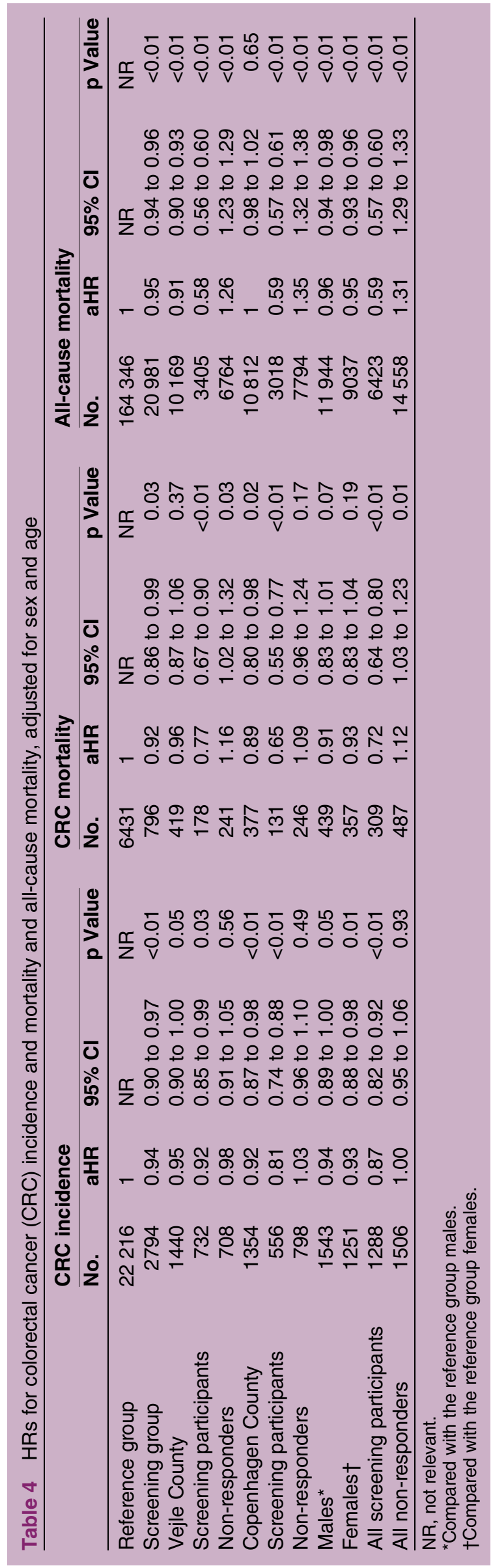


Sensitivity analyses to assess the influence of 3757 persons, who were excluded from screening in the feasibility study for unknown reasons, showed that their inclusion in the analyses did not influence our results significantly (data not shown).

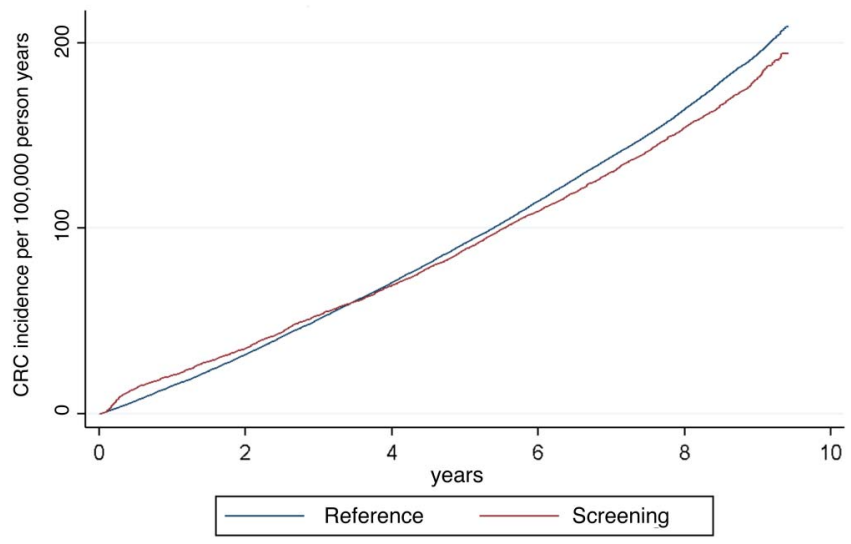

Figure 2 Cumulative colorectal cancer incidence in persons from Vejle and Copenhagen counties invited to screening and in persons from the rest of Denmark.

\section{COPENHAGEN COUNTY}

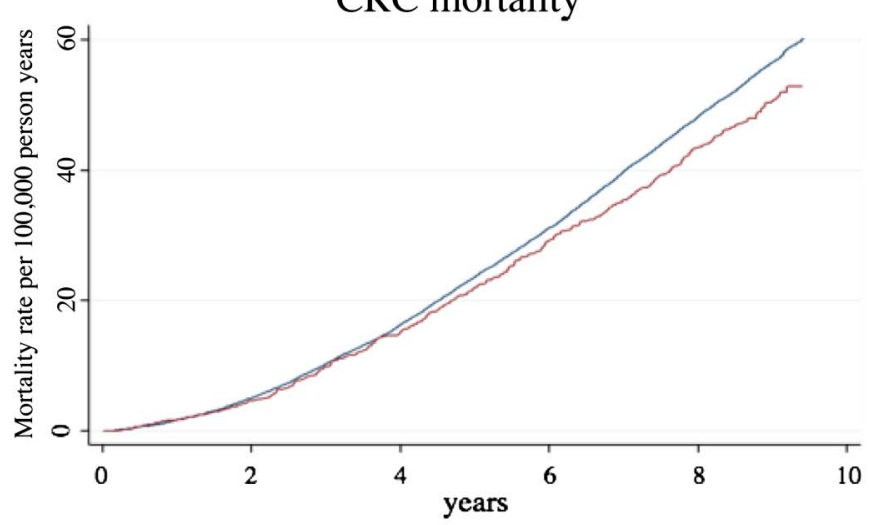

All-cause mortality

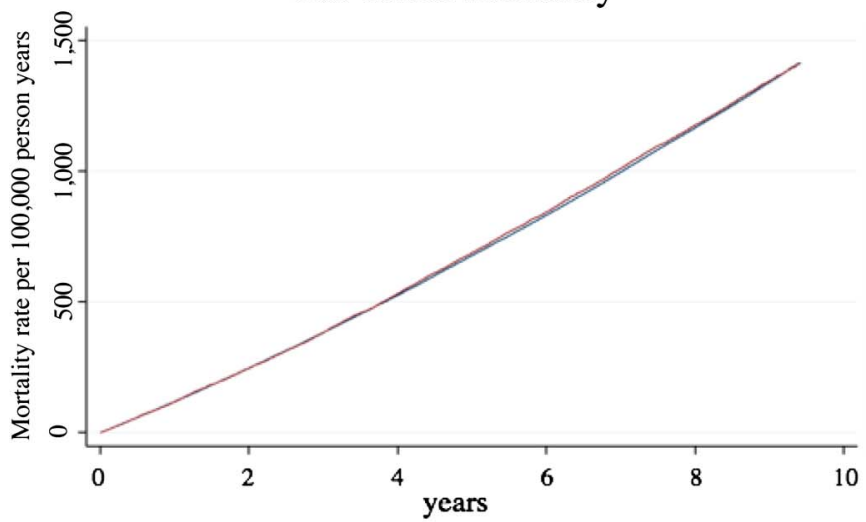

\section{DISCUSSION}

Approximately 10 years after a single round of gFOBT screening for CRC, we found a significant $8 \%$ lower incidence-based CRC mortality in the invited screening group than in the reference group of other Danes. This could point to a beneficial impact of the screening. It should, however, be taken into account that the invited screening group did not represent a random sample of the Danish population. The all-cause mortality was $5 \%$ lower in the invited screening group than in the reference group. As the difference in the deficits of $8 \%$ for CRC mortality and $5 \%$ for all-cause mortality is marginal, it is not possible to attribute the reduced CRC mortality to one screening round alone. The uncertainty in these numbers came from the Vejle part of the invited screening group, as this group clearly represented a healthier subpopulation than the rest of Danes. One may argue that the Copenhagen part of the invited screening group would give a clearer picture of the screening effect as this group had a $11 \%$ deficit in CRC mortality and an all-cause mortality similar to that of other Danes. This is, however, a subgroup analysis not originally planned for.

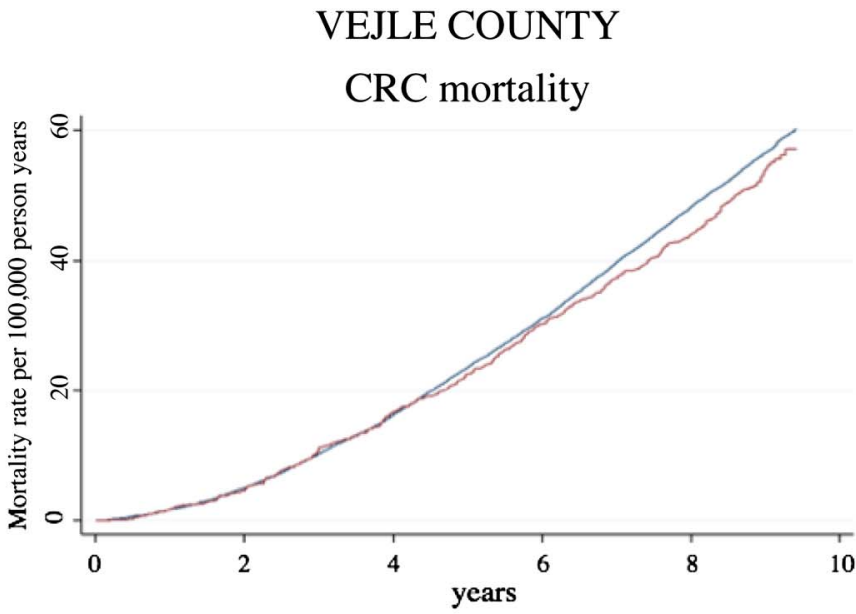

All-cause mortality

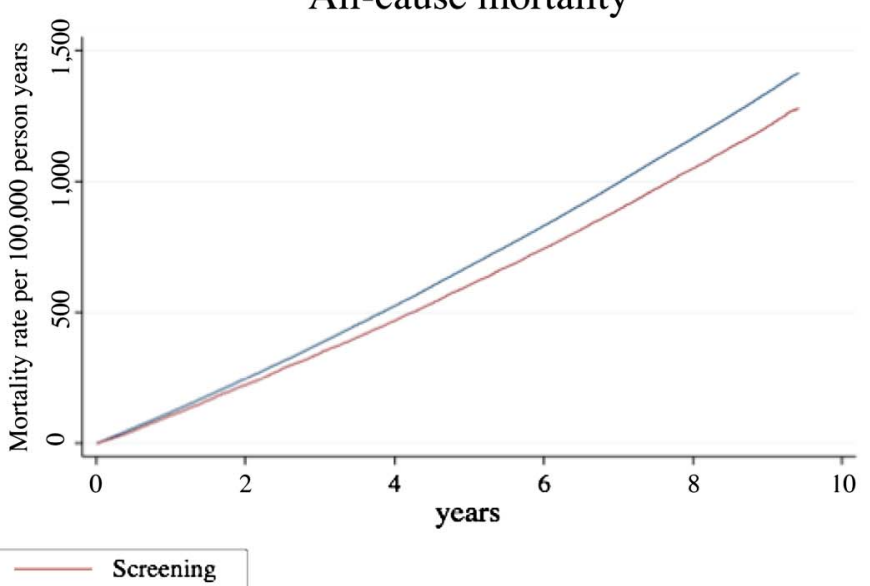

Figure 3 Cumulative colorectal cancer mortality and all-cause mortality in persons in Vejle and Copenhagen counties invited to screening and in persons in the rest of Denmark. 
Screening participants represented a selected group. When compared with the reference group, participants had a $41 \%$ lower all-cause mortality, while nonparticipants had a $31 \%$ higher all-cause mortality. This highlights the problem of inequitable uptake of screening services; those who participate are healthier than those who do not. Since we could not control for selective participation in the HRs for CRC mortality, the reported deficit in CRC mortality among participants should be interpreted with caution.

When compared with the earlier randomised controlled trial on gFOBT CRC screening in Funen, Denmark, the participation in the feasibility study was low, $49 \%$ when compared with $67 \%$, though the CRC detection rates were identical, 1.9 per 1000 participants in the feasibility study and 1.8 in the trial. ${ }^{5}$ Within the group invited to screening, $18 \%$ had at least one colonoscopy during the $\sim 10$ years of follow-up. Colonoscopy rate was higher in the screening group within the first 2 years, but equal to findings in the reference group the following years. This may be due to cancer awareness within the general population and among general practitioners in Denmark.

The screen-detected cases represented only 155 out of the 2794 CRC cases in the group invited to screening. The remaining 2639 CRC cases were detected within the routine healthcare system with prognosis comparable to cases detected in the reference group. The polypectomy rate within the first 2 years after invitation was higher in the screening group than in the reference group. Colorectal adenomas may be cancer precursors, ${ }^{11}$ and there is evidence that detection and removal of these lesions may prevent cancers. ${ }^{12}$ This is expected to have contributed to the slightly lower CRC incidence in the screening group than in the reference group; 2794 observed cases vs $2972(=2794 / 0.94$ aHR for CRC incidence) expected cases, resulting in a deficit of 178 cases. The potential effect of the one-round screening on the CRC mortality was therefore based on the relatively few cases detected at screening or prevented due to polypectomy. This finding clearly illustrates the need for repeated testing in faecal occult blood-based screening programmes.

van Leeuwen et $a l^{10}$ have shown that, in prostate cancer screening, disease-specific mortality has been systematically underestimated due to risk of misclassification of cause-of-death. The authors argue that estimation of the excess mortality is of additional value to a diseasespecific mortality analysis since it measures the effect of screening in the presence of all competing risks. We did not notice any discrepancy between disease-specific mortality and excess mortality in our data, which indicates high data quality and accuracy.

It was a strength of this study that we could individually follow all persons invited to screening in 2005/2006 for a period of $\sim 10$ years. It was furthermore a strength that linkage to the Danish Cancer Register allowed us to calculate incidence-based CRC mortality. It was a weakness of this observational study that we were not able to control for prescreening differences in health status between the screening counties and the rest of Denmark. This is in contrast to the Danish studies on the effect of mammography screening on breast cancer mortality, ${ }^{13}{ }^{14}$ where calendar period and region were controlled for in the analysis. The study relied on health service register data submitted by health providers. While the quality of register data is considered to be high, ${ }^{15}$ we did encounter some discrepancies in the register data on colonoscopies and polypectomies, which may have introduced bias.

Biennial gFOBT CRC screening has been introduced in many countries based on the results from the randomised controlled trials in Minnesota, ${ }^{2}$ Göteborg, ${ }^{3}$ Nottingham ${ }^{4}$ and Funen. ${ }^{5}$ A systematic review from the US Preventive Services Task Force showed a significant reduction in CRC mortality after 11-30 years (relative risk (RR) $0.91,95 \%$ CI 0.84 to 0.98 ) and after 30 years (RR $0.78,95 \%$ CI 0.65 to 0.93$).{ }^{7}$ A Finnish populationbased gFOBT CRC screening programme was introduced as a public health trial, where a random $50 \%$ of each birth cohort was invited. ${ }^{6}$ The results of the Finnish study were too new to be included in the US systematic review. However, after accumulation of 180000 persons invited to screening, and with a follow-up of on average 4.5 years, there was no impact of the screening on CRC mortality (RR $1.04,95 \%$ CI 0.84 to 1.28 ). ${ }^{6}$ In contrast to the Finnish study, we did not observe any difference in screening effect between men and women. A 4.5 average years of follow-up is a short time, but the Finnish results, together with the more limited data from this Danish feasibility study, nevertheless indicate that close monitoring of the outcome of the ongoing CRC screening programmes is warranted. The combined effect of increased cancer awareness, good access to colonoscopy and accelerated cancer care pathways may improve early detection and treatment for CRC and diminish the potential impact of gFOBT screening. The FIT is more acceptable for participants than the gFOBT, and it also has higher sensitivity for faecal blood. ${ }^{16}{ }^{17}$ For this reason, many countries, including Denmark, now use FIT in their CRC screening programmes.

\section{CONCLUSION}

An 10 years' follow-up of a one-round CRC screening in a Danish feasibility study showed an $8 \%$ deficit in CRC mortality when compared with Danes in the rest of the country. There was however almost the same deficit in the all-cause mortality. On this basis, it is not possible to conclude that screening in the feasibility study had an effect on CRC mortality. Our results, together with new results from the large Finnish CRC screening programme, indicate that close monitoring of the outcome of CRC screening programmes is warranted.

Acknowledgements The authors thank Søren Nymand Lophaven for statistical advice. 
Contributors All authors were involved in conceptualising the study and approved the final version of the manuscript. $A B$ and $E L$ designed the study and statistical analyses. $A B$ and $J L$ collected data. $A B$ cleaned and analysed the data. $A B$ and $E L$ drafted the paper. All authors critically revised the manuscript.

Funding This research was supported by a grant of 25000 DKK from Copenhagen University Hospital, Herlev, to cover data retrieval from the Danish Health Registers. The research received no other funding from the public, commercial or not-for-profit sectors.

Competing interests None declared.

Provenance and peer review Not commissioned; externally peer reviewed.

Data sharing statement No additional data are available.

Open Access This is an Open Access article distributed in accordance with the Creative Commons Attribution Non Commercial (CC BY-NC 4.0) license, which permits others to distribute, remix, adapt, build upon this work noncommercially, and license their derivative works on different terms, provided the original work is properly cited and the use is non-commercial. See: http:// creativecommons.org/licenses/by-nc/4.0/

\section{REFERENCES}

1. NORDCAN. 2015. (cited 4 February 2015). http://www-dep.iarc.fr/ NORDCAN/DK/frame.asp

2. Mandel JS, Bond JH, Church TR, et al. Reducing mortality from colorectal cancer by screening for fecal occult blood. Minnesota Colon Cancer Control Study. N Engl J Med 1993;328:1365-71.

3. Lindholm E, Brevinge $\mathrm{H}$, Haglind $\mathrm{E}$. Survival benefit in a randomized clinical trial of faecal occult blood screening for colorectal cancer. Br J Surg 2008;95:1029-36.

4. Hardcastle JD, Chamberlain JO, Robinson MH, et al. Randomised controlled trial of faecal-occult-blood screening for colorectal cancer. Lancet 1996;348:1472-7.
5. Kronborg O, Fenger C, Olsen J, et al. Randomised study of screening for colorectal cancer with faecal-occult-blood test. Lancet 1996;348:1467-71.

6. Pitkäniemi J, Seppa K, Hakamä M, et al. Effectiveness of screening for colorectal cancer with a faecal occult-blood test, in Finland. BMJ Open Gastroenterol 2015;2:e000034.

7. Lin JS, Piper MA, Perdue LA, et al. Screening for colorectal cancer: updated evidence report and systematic review for the US Preventive Services Task Force. JAMA 2016;315:2576-94.

8. Kræftens Bekæmpelse (Danish Cancer society). Feasibility study of screening for colorectal cancer in Copenhagen County. 2007. https:// www.cancer.dk/dyn/resources/File/file/6/556/1385260913/ samletslutrapport.pdf [In Danish].

9. Bisgaard C, Jakobsen C, Bliksted J, et al. Screening for colorectal cancer in Vejle Amt - a feasibility study. 2007. https://www.cancer. dk/dyn/resources/File/file/7/557/1385260913/screeningtarmkrft_ tilibenholten.pdf [In Danish].

10. van Leeuwen PJ, Kranse R, Hakulinen T, et al. Disease-specific mortality may underestimate the total effect of prostate cancer screening. J Med Screen 2010;17:204-10.

11. Muto T, Bussey HJ, Morson BC. The evolution of cancer of the colon and rectum. Cancer 1975;36:2251-70.

12. Zauber AG, Winawer SJ, O'Brien MJ, et al. Colonoscopic polypectomy and long-term prevention of colorectal-cancer deaths. N Engl J Med 2012;366:687-96.

13. Njor SH, Schwartz W, Blichert-Toft M, et al. Decline in breast cancer mortality: how much is attributable to screening? J Med Screen 2015;22:20-7.

14. Olsen AH, Njor SH, Vejborg I, et al. Breast cancer mortality in Copenhagen after introduction of mammography screening: cohort study. BMJ 2005;330:220.

15. Schmidt M, Schmidt SA, Sandegaard JL, et al. The Danish National Patient Registry: a review of content, data quality, and research potential. Clin Epidemiol 2015;7:449-90.

16. Hol L, Wilschut JA, van Ballegooijen M, et al. Screening for colorectal cancer: random comparison of guaiac and immunochemical faecal occult blood testing at different cut-off levels. Br J Cancer 2009;100:1103-10.

17. van Rossum LG, van Rijn AF, Laheij RJ, et al. Random comparison of guaiac and immunochemical fecal occult blood tests for colorectal cancer in a screening population. Gastroenterology 2008;135:82-90. 